\title{
Strings of length 3 in Grand-Dyck paths and the Chung-Feller property
}

\author{
K. Manes, A. Sapounakis, I. Tasoulas and P. Tsikouras \\ Department of Informatics \\ University of Piraeus, Piraeus, Greece \\ $\{$ kmanes, arissap, jtas, pgtsik\}@unipi.gr
}

Submitted: Feb 9, 2012; Accepted: Mar 28, 2012; Published: Apr 7, 2012

Mathematics Subject Classifications: 05A15, 05A19

\begin{abstract}
This paper deals with the enumeration of Grand-Dyck paths according to the statistic "number of occurrences of $\tau$ " for every string $\tau$ of length 3 , taking into account the number of flaws of the path. Consequently, some new refinements of the Chung-Feller theorem are obtained.
\end{abstract}

\section{Introduction}

Throughout this paper, a path is considered to be a lattice path on the integer plane, consisting of steps $u=(1,1)$ (called rises) and $d=(1,-1)$ (called falls). Since the sequence of steps of a path is encoded by a word in $\{u, d\}^{*}$, we will make no distinction between these two notions. The length of a path is the number of its steps.

A Grand-Dyck path is a path that starts and ends at the same height. It is convenient to consider that the starting point of a Grand-Dyck path is the origin of a pair of axes. Obviously, every Grand-Dyck path ends at a point $(0,2 n)$ where $n$ is referred to as the semilength of the path.

The set of Grand-Dyck paths of semilength $n$ is denoted by $\mathcal{G}_{n}$, and we set $\mathcal{G}=\bigcup_{n=0}^{\infty} \mathcal{G}_{n}$, where $\mathcal{G}_{0}=\{\varepsilon\}$ and $\varepsilon$ is the empty path.

Every rise of a path $\alpha$ which lies below (resp. above) the $x$-axis is called a flaw (resp. a non-flaw) of $\alpha$. The number of flaws of $\alpha$ is denoted by $p(\alpha)$.

The set of Grand-Dyck paths of semilength $n$ having $m$ flaws is denoted $\mathcal{G}_{n, m}$. In particular, we set $\mathcal{D}_{n}=\mathcal{G}_{n, 0}$ (resp. $\overline{\mathcal{D}}_{n}=\mathcal{G}_{n, n}$ ) the set of Dyck paths (resp. inverted Dyck paths). It is well known that the set $\mathcal{D}_{n}$ is counted by the Catalan number $C_{n}=\frac{1}{n+1}\left(\begin{array}{c}2 n \\ n\end{array}\right)$ (see A000108 in [22]). Furthermore, the classical Chung-Feller theorem [5,12] states that $\left|\mathcal{G}_{n, m}\right|=C_{n}$, for all $m \in[0, n]$. 
Every $\alpha \in \mathcal{G} \backslash \overline{\mathcal{D}}$ can be uniquely decomposed in the form $a=\beta u \gamma d \delta$ where $\beta \in \overline{\mathcal{D}}$, $\gamma \in \mathcal{D}$ and $\delta \in \mathcal{G}$ (see Figure $1 \mathrm{i}$ ); this decomposition will be referred to as the first nonflaw decomposition and will be used in the sequel extensively. Similarly, the decomposition $\alpha=\delta d \beta u \gamma$ of $\mathcal{G} \backslash \mathcal{D}$ will be referred to as the last flaw decomposition (see Figure 1 ii).

A path $\tau \in\{u, d\}^{*}$, called in this context string, occurs in a path $\alpha$ if $\alpha=\beta \tau \gamma$, for some $\beta, \gamma \in\{u, d\}^{*}$. The number of occurrences of the string $\tau$ in $\alpha$, is denoted by $|\alpha|_{\tau}$. Given a string $\tau$, the symmetric string of $\tau$ with respect to a horizontal (resp. vertical) axis is denoted by $\bar{\tau}$ (resp. $\tau^{\prime}$ ).

A wide range of articles dealing with the occurrence of strings in Dyck paths appear frequently in the literature $[1-4,6-10,13-21,23]$. For the occurrences of strings in GrandDyck paths it is interesting to take also into account the number of flaws of the paths. In this direction Ma and Yeh [11] have studied the statistic "number of occurrences of $\tau$ " in Grand-Dyck paths for all strings $\tau$ of length 2. In this work, the same subject is studied for all strings $\tau$ of length 3 obtaining some new Chung-Feller type results.

For this we use the generating function

$$
F_{\tau}(x, y, z)=\sum_{\alpha \in \mathcal{G}} x^{|\alpha|_{\tau}} y^{p(\alpha)} z^{|\alpha|_{u}} .
$$

Clearly, using the two classical bijections of $\mathcal{G}$ according to which every $a \in \mathcal{G}$ is mapped to $\bar{\alpha}$ and $\alpha^{\prime}$, we obtain that

$$
\left[x^{k} y^{m} z^{n}\right] F_{\tau}=\left[x^{k} y^{n-m} z^{n}\right] F_{\bar{\tau}}=\left[x^{k} y^{m} z^{n}\right] F_{\tau^{\prime}} \text {, where } m \leqslant n .
$$

Thus, among the eight strings of length 3 it is enough to restrict ourselves to the following three: $u u u, u d u, d u u$.

In [11], it has been proved, both analytically and bijectively, that the number of GrandDyck paths with semilength $n$, having $m$ flaws and $k$ occurrences of the string $\tau=u^{2}$, is independent of $m$, thus satisfying the Chung-Feller property. In fact, the following generalization holds.

Proposition 1.1. The number of Grand-Dyck paths with semilength $n$, having $m$ flaws and $k_{i}$ occurrences of the string $u^{i}$, for every $i \geqslant 2$, is independent of $m$.

Although the previous proposition can be proved analytically, it is easier to be justified by using the simple mapping $\phi: \mathcal{G} \backslash \overline{\mathcal{D}} \rightarrow \mathcal{G} \backslash \mathcal{D}$ with $\phi(\beta u \gamma d \delta)=\delta d \beta u \gamma$, where $\beta \in \bar{D}$, $\gamma \in \mathcal{D}$, and $\delta \in \mathcal{G}$, which is a length-preserving bijection that increases the number of flaws by one and preserves the number of occurrences of the strings $u^{i}$; (see Figure 1).

In particular, the number of Grand-Dyck paths with semilength $n$, having $m$ flaws and $k$ occurrences of the string $u^{3}$ is counted by the sequence A092107 in [22] and it is given by the complex formula [19]

$$
\frac{1}{n+1} \sum_{j=0}^{k}(-1)^{k-j}\left(\begin{array}{c}
n+j \\
n
\end{array}\right)\left(\begin{array}{c}
n+1 \\
k-j
\end{array}\right) \sum_{i=j}^{[(n+j) / 2]}\left(\begin{array}{c}
n+j+1-k \\
i+1
\end{array}\right)\left(\begin{array}{c}
n-i \\
i-j
\end{array}\right) .
$$

In the following two sections we will study the strings $u d u$ and $d u u$. 


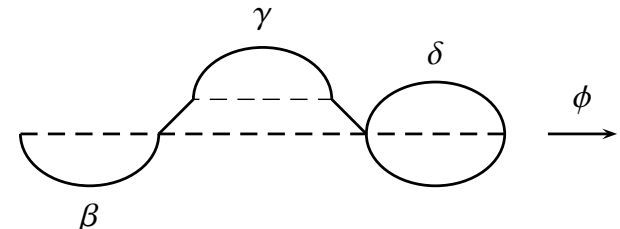

i The first non-flaw decomposition of $\mathcal{G} \backslash \overline{\mathcal{D}}$

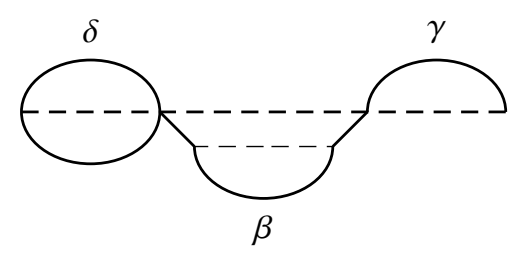

ii The last flaw decomposition of $\mathcal{G} \backslash \mathcal{D}$

Figure 1: The bijection $\phi$

\section{The string $\tau=u d u$}

Let $F(x, y, z)=\sum_{a \in \mathcal{G}} x^{|a|_{u d u}} y^{p(a)} z^{|a|_{u}}=\sum_{m \geqslant 0} f_{m}(x, z) y^{m}$, where $f_{m}(x, z)$ is the generating function of the set of Grand-Dyck paths with $m$ flaws, with respect to the number of occurrences of $\tau=u d u$ and to the semilength.

The Dyck path statistic "number of occurrences of $u d u$ " has been studied independently by Sun [23] and Merlini, Sprungoli and Verri [17] where it is stated that the corresponding generating function $f_{0}$ satisfies the equation

$$
z f_{0}^{2}(x, z)=(1-(x-1) z)\left(f_{0}(x, z)-1\right)
$$

and has coefficients

$$
\left[x^{k} z^{n}\right] f_{0}= \begin{cases}1, & k=n=0 \\
\left(\begin{array}{c}
n-1 \\
k
\end{array}\right) M_{n-k-1}, & k \in[0, n-1],\end{cases}
$$

where $M_{n}$ is the $n$-th Motzkin number (see A001006 in [22]).

\section{Theorem 2.1.}

$$
f_{m}(x, z)=(1-(x-1) z) \sum_{n \geqslant m} \sum_{k=0}^{n-1}\left(\begin{array}{c}
n-1 \\
k
\end{array}\right) M_{n-k-1} x^{k} z^{n}, \quad m \geqslant 1 .
$$

Proof. We will first show that

$$
F(x, y, z)=\frac{(1-(x-1) z) f_{0}(x, y z)}{1-(x-1) z-z f_{0}(x, y z) f_{0}(x, z)} .
$$

For every $\alpha \in \mathcal{G} \backslash \bar{D}$ with $a=\beta u \gamma d \delta, \beta \in \overline{\mathcal{D}}, \gamma \in \mathcal{D}, \delta \in \mathcal{G}$ we have that

$$
|\alpha|_{\tau}=|\beta|_{\tau}+|\gamma|_{\tau}+|\delta|_{\tau}+[\gamma=\varepsilon][\delta \in \mathcal{A}]
$$

where $\mathcal{A}=\{a \in \mathcal{G}: a$ starts with $u\}$, and $[P]$ is the Iverson notation: $[P]=1$ if $P$ is true and $[P]=0$ if $P$ is false. 
From the previous equality, using the first non-flaw decomposition of $\mathcal{G} \backslash \bar{D}$, it follows that

$$
\begin{aligned}
& F(x, y, z) \\
& =\sum_{\beta \in \overline{\mathcal{D}}} x^{|\beta|_{\tau}} y^{p(\beta)} z^{|\beta|_{u}}+z \sum_{\beta \in \overline{\mathcal{D}}} x^{|\beta|_{\tau}} y^{p(\beta)} z^{|\beta|_{u}} \sum_{\substack{\gamma \in \mathcal{D} \\
\delta \in \mathcal{G}}} x^{|\gamma|_{\tau}+|\delta|_{\tau}+[\gamma=\varepsilon][\delta \in \mathcal{A}]} y^{p(\delta)} z^{|\gamma|_{u}+|\delta|_{u}} \\
& =\sum_{\beta \in \mathcal{D}} x^{|\beta|_{\bar{\tau}}}(y z)^{|\beta|_{u}}+z \sum_{\beta \in \mathcal{D}} x^{|\beta|_{\bar{\tau}}}(y z)^{|\beta|_{u}}\left(\sum_{\substack{\gamma \in \mathcal{D} \backslash\{\varepsilon\} \\
\delta \in \mathcal{G}}} x^{|\gamma|_{\tau}+|\delta|_{\tau}} y^{p(\delta)} z^{|\gamma|_{u}+|\delta|_{u}}+\sum_{\delta \in \mathcal{G}} x^{|\delta|_{\tau}+[\delta \in A]} y^{p(\delta)} z^{|\delta|_{u}}\right) \\
& =f_{0}(x, y z)\left(1+z\left(f_{0}(x, z)-1\right) F(x, y, z)+z(F(x, y, z)+(x-1) A(x, y, z))\right),
\end{aligned}
$$

where $A(x, y, z)$ is the generating function of the set $\mathcal{A}$.

It follows that

$$
F(x, z, y)=f_{0}(x, y z)\left(1+z f_{0}(x, z) F(x, y, z)+z(x-1) A(x, y, z)\right) .
$$

Every $a \in \mathcal{A}$ can be written uniquely in the form $a=u \gamma d \delta$, where $\gamma \in \mathcal{D}, \delta \in \mathcal{G}$, so that $|a|_{\tau}=|\gamma|_{\tau}+|\delta|_{\tau}+[\gamma=\varepsilon][\delta \in \mathcal{A}]$, giving

$$
A(x, y, z)=z\left(\left(f_{0}(x, z)-1\right) F(x, y, z)+F(x, y, z)+(x-1) A(x, y, z)\right) .
$$

Hence,

$$
A(x, y, z)=\frac{z f_{0}(x, z) F(x, y, z)}{1-(x-1) z} .
$$

By substituting the previous expression of $A(x, y, z)$ in relation (2.4), we obtain the required relation $(2.3)$.

From relation (2.3), using relation (2.1), we have that

$$
\begin{aligned}
& F(x, y, z)-f_{0}(x, z) \\
& =\frac{(1-(x-1) z)\left(f_{0}(x, y z)-f_{0}(x, z)+f_{0}(x, y z)\left(f_{0}(x, z)-1\right)\right)}{1-(x-1) z-z f_{0}(x, y z) f_{0}(x, z)} \\
& =\frac{y(1-(x-1) z) f_{0}(x, z)\left(f_{0}(x, y z)-1\right)\left(f_{0}(x, z)-f_{0}(x, y z)\right)}{y(1-(x-1) z)\left(f_{0}(x, z)-f_{0}(x, y z)\right)-y(1-(x-1) z)\left(f_{0}(x, z)-1\right) f_{0}(x, y z)+(1-(x-1) y z)\left(f_{0}(x, y z)-1\right) f_{0}(x, z)} \\
& =\frac{y(1-(x-1) z) f_{0}(x, z)\left(f_{0}(x, y z)-1\right)\left(f_{0}(x, z)-f_{0}(x, y z)\right)}{(1-y) f_{0}(x, z)\left(f_{0}(x, y z)-1\right)} .
\end{aligned}
$$

Thus, we have that

$$
F(x, y, z)=f_{0}(x, z)+(1-(x-1) z)\left(f_{0}(x, z)-f_{0}(x, y z)\right) \frac{y}{1-y} .
$$

From relation (2.2), we have that

$$
\left[y^{m}\right]\left(f_{0}(x, z)-f_{0}(x, y z)\right)= \begin{cases}f_{0}(x, z)-1, & m=0 \\
-\sum_{k=0}^{m-1}\left(\begin{array}{c}
m-1 \\
k
\end{array}\right) M_{m-k-1} x^{k} z^{m}, & m \geqslant 1 .\end{cases}
$$


Hence, from relation (2.5), we obtain that, for $m \geqslant 1$,

$$
\begin{aligned}
f_{m}(x, z) & =(1-(x-1) z)\left[y^{m-1}\right] \frac{f_{0}(x, z)-f_{0}(x, y z)}{1-y} \\
& =(1-(x-1) z)\left(f_{0}(x, z)-1-\sum_{n=1}^{m-1} \sum_{k=0}^{n-1}\left(\begin{array}{c}
n-1 \\
k
\end{array}\right) M_{n-k-1} x^{k} z^{n}\right) \\
& =(1-(x-1) z) \sum_{n \geqslant m} \sum_{k=0}^{n-1}\left(\begin{array}{c}
n-1 \\
k
\end{array}\right) M_{n-k-1} x^{k} z^{n}
\end{aligned}
$$

Corollary 2.2. The number of Grand-Dyck paths with semilength $n$, having $m$ flaws and $k$ occurrences of the string udu is equal to

$$
\left[x^{k} z^{n}\right] f_{m}= \begin{cases}\left(\begin{array}{c}
n-1 \\
k
\end{array}\right) M_{n-k-1}, & m=0, n \\
\left(\begin{array}{c}
n-2 \\
k
\end{array}\right)\left(M_{n-k-1}+M_{n-k-2}\right), & m \in[1, n-1] .\end{cases}
$$

Proof. By Theorem 2.1, for $m \in[1, n-1]$, we have that

$$
\begin{aligned}
{\left[x^{k} z^{n}\right] f_{m} } & =\left(\begin{array}{c}
n-1 \\
k
\end{array}\right) M_{n-k-1}-\left(\begin{array}{c}
n-2 \\
k-1
\end{array}\right) M_{n-k-1}+\left(\begin{array}{c}
n-2 \\
k
\end{array}\right) M_{n-k-2} \\
& =\left(\begin{array}{c}
n-2 \\
k
\end{array}\right) M_{n-k-1}+\left(\begin{array}{c}
n-2 \\
k
\end{array}\right) M_{n-k-2} \\
& =\left(\begin{array}{c}
n-2 \\
k
\end{array}\right)\left(M_{n-k-1}+M_{n-k-2}\right) .
\end{aligned}
$$

The cases where $m=0, n$ are obvious and are omitted.

Corollary 2.3. The number of Grand-Dyck paths with semilength $n$, having $k$ occurrences of the string udu is equal to

$$
\left(\begin{array}{c}
n-1 \\
k
\end{array}\right)\left((n-k+1) M_{n-k-1}+(n-k-1) M_{n-k-2}\right) .
$$

For further information concerning the double sequence described in the previous corollary, see A097692 in [22].

Remark. Corollary 2.2 is a Chung-Feller type theorem, since it shows that the number of Grand-Dyck paths with semilength $n$ having $m$ flaws and $k$ occurrences of the string $u d u$ is independent of $m$, for $m \in[1, n-1]$.

We end this section by giving a combinatorial proof of this result. For this purpose it is enough to construct a bijection from $\mathcal{G}_{n, m}$ to $\mathcal{G}_{n, m+1}$ which preserves the number of occurrences of the string $u d u$, for every $m \in[1, n-2]$.

We observe that every $a \in \mathcal{G} \backslash \overline{\mathcal{D}}$ (resp. $a \in \mathcal{G} \backslash \mathcal{D}$ ) can be written uniquely in the form $\alpha=\beta_{1} u \gamma_{1} d \beta_{2} \delta \gamma_{2}$ (resp. $\alpha=\beta_{1} \delta \gamma_{1} d \beta_{2} u \gamma_{2}$ ) where $\beta_{1}, \beta_{2} \in \overline{\mathcal{D}}, \gamma_{1}, \gamma_{2} \in \mathcal{D}, \delta \in \mathcal{G}$ such that $\delta=\varepsilon$ or $\delta$ starts and ends with $u$. 
The mapping $\psi: \mathcal{G} \backslash \overline{\mathcal{D}} \rightarrow \mathcal{G} \backslash \mathcal{D}$ with

$$
\psi\left(\beta_{1} u \gamma_{1} d \beta_{2} \delta \gamma_{2}\right)=\beta_{1} \delta \gamma_{1} d \beta_{2} u \gamma_{2}
$$

(see Figure 2) is a length-preserving bijection that increases the number of flaws by one.

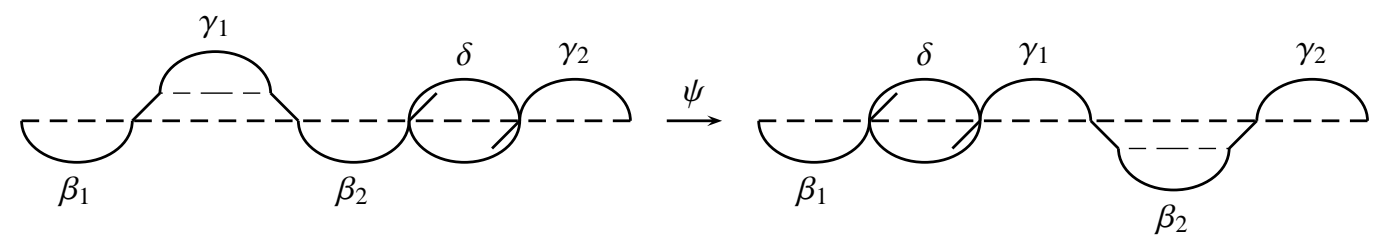

Figure 2: The bijection $\psi$

Furthermore, $|\psi(\alpha)|_{u d u}=|\alpha|_{u d u}$ for every $\alpha \in \mathcal{G} \backslash \overline{\mathcal{D}}$ with $1 \leqslant p(\alpha) \leqslant|a|_{u}-2$.

Indeed, every occurrence of $\tau$ in $\alpha$ (resp. in $\psi(\alpha)$ ) that does not lie entirely in one of $\beta_{1}, \beta_{2}, \gamma_{1}, \gamma_{2}$ exists if and only if $\gamma_{1}=\beta_{2}=\varepsilon$ and $\delta \gamma_{2} \neq \varepsilon\left(\operatorname{resp} . \gamma_{1}=\beta_{2}=\varepsilon\right.$ and $\left.\beta_{1} \delta \neq \varepsilon\right)$.

Then, if $\delta \neq \varepsilon$, the required equality is obviously true. On the other hand, if $\delta=\varepsilon$, since $|\alpha|_{u}-p(\alpha) \geqslant 2$ (resp. $p(\alpha) \geqslant 1$ ), it follows that, if $\gamma_{1}=\varepsilon$ (resp. $\beta_{2}=\varepsilon$ ), then $\gamma_{2} \neq \varepsilon$ (resp. $\left.\beta_{1} \neq \varepsilon\right)$. Hence, the required equality holds in this case, too.

Thus, the restriction of $\psi$ on the set $\mathcal{G}_{n, m}$ gives the required bijection.

\section{$3 \quad$ The string $\tau=d u u$}

Let $F(x, y, z)=\sum_{a \in \mathcal{G}} x^{|a|_{\text {duu }}} y^{p(a)} z^{|a|_{u}}=\sum_{m \geqslant 0} f_{m}(x, z) y^{m}$, where $f_{m}(x, z)$ is the generating function of the set of Grand-Dyck paths with $m$ flaws, with respect to the number of occurrences of $\tau=d u u$ and to the semilength.

Let $g_{0}(x, z)$ be the generating function of $\mathcal{D}$ with respect to the number of occurrences of $\bar{\tau}=u d d$ and to the semilength. The Dyck path statistics "number of occurrences of $d u u$ " and "number of occurences of $u d d$ " have been studied by Deutsch [9] and Sapounakis, Tasoulas, Tsikouras [19] respectively, where it is stated that the corresponding generating functions $f_{0}, g_{0}$ satisfy the equations

$$
x z f_{0}^{2}(x, z)-(1+2(x-1) z) f_{0}(x, z)+1+(x-1) z=0
$$

and

$$
z(1+(x-1) z) g_{0}^{2}(x, z)-g_{0}(x, z)+1=0
$$

with coefficients

$$
a_{n, k}=\left[x^{k} z^{n}\right] f_{0}= \begin{cases}1, & k=n=0 \\
2^{n-2 k-1} C_{k}\left(\begin{array}{c}
n-1 \\
2 k
\end{array}\right), & n \geqslant 1\end{cases}
$$


and

$$
b_{n, k}=\left[x^{k} z^{n}\right] g_{0}= \begin{cases}1, & k=0 \\
\frac{1}{n+1}\left(\begin{array}{c}
n+1 \\
k
\end{array}\right) \sum_{j=2 k}^{n}\left(\begin{array}{c}
j-k-1 \\
k-1
\end{array}\right)\left(\begin{array}{c}
n+1-k \\
n-j
\end{array}\right), & k \geqslant 1 .\end{cases}
$$

From relations (3.1), (3.2) it follows that

$$
f_{0}(x, z)=\frac{1+2(x-1) z-\sqrt{\Delta}}{2 x z} \text { and } g_{0}(x, z)=\frac{1-\sqrt{\Delta}}{2 z(1+(x-1) z)},
$$

where $\Delta=1-4 z-4 z^{2}(x-1)$.

From the previous two equalities it follows that

$$
f_{0}(x, z)=\left(1-(x-1) z\left(f_{0}(x, z)-1\right)\right) g_{0}(x, z)=\frac{1}{1-z g_{0}(x, z)} .
$$

\section{Theorem 3.1.}

$$
f_{m}(x, z)=\left(1-(x-1) z\left(f_{0}(x, z)-1\right)\right) \sum_{n \geqslant m} \sum_{k \geqslant 0} b_{n, k} x^{k} z^{n}, \quad m \geqslant 0 .
$$

Proof. We will first show that

$$
F(x, y, z)=\frac{g_{0}(x, y z)}{1-z(1+(x-1) y z) g_{0}(x, z) g_{0}(x, y z)} .
$$

For every $\alpha \in \mathcal{G} \backslash \bar{D}$ with $a=\beta u \gamma d \delta, \beta \in \overline{\mathcal{D}}, \gamma \in \mathcal{D}, \delta \in \mathcal{G}$ we have that

$$
|\alpha|_{\tau}=|\beta|_{\tau}+|\gamma|_{\tau}+|\delta|_{\tau}+[\beta \text { ends with } d u]+[\delta \in \mathcal{A}]
$$

where $\mathcal{A}=\left\{a \in \mathcal{G}: a\right.$ starts with $\left.u^{2}\right\}$.

From the previous equality, using the first non-flaw decomposition of $\mathcal{G} \backslash \bar{D}$, it follows that

$$
\begin{aligned}
F(x, y, z) & =\sum_{\beta \in \overline{\mathcal{D}}} x^{|\beta|_{\tau}} y^{p(\beta)} z^{|\beta|_{u}} \\
& +z \sum_{\beta \in \overline{\mathcal{D}}} x^{|\beta|_{\tau}+[\beta \text { ends with } d u]} y^{p(\beta)} z^{|\beta|_{u}} \sum_{\gamma \in \mathcal{D}} x^{|\gamma|_{\tau}} z^{|\gamma|_{u}} \sum_{\delta \in \mathcal{G}} x^{|\delta|_{\tau}+[\delta \in \mathcal{A}]} y^{p(\delta)} z^{|\delta|_{u}} \\
= & g_{0}(x, y z)+z\left(g_{0}(x, y z)+(x-1) \sum_{\substack{\beta \in \overline{\mathcal{D}} \\
\beta \text { ends with } d u}} x^{|\beta|_{\tau}}(y z)^{|\beta|_{u}}\right) f_{0}(x, z)(F(x, y, z) \\
& +(x-1) A(x, y, z)) \quad
\end{aligned}
$$

where $A(x, y, z)$ is the generating function of the set $\mathcal{A}$.

It follows that

$$
F(x, y, z)=g_{0}(x, y z)\left(1+z(1+(x-1) y z) f_{0}(x, z)(F(x, y, z)+(x-1) A(x, y, z))\right) .
$$


Every $a \in \mathcal{A}$ can be written uniquely in the form $a=u \gamma d \delta$, where $\gamma \in \mathcal{D} \backslash\{\varepsilon\}, \delta \in \mathcal{G}$, so that $|a|_{\tau}=|\gamma|_{\tau}+|\delta|_{\tau}+[\delta \in \mathcal{A}]$, giving

$$
A(x, y, z)=z\left(f_{0}(x, z)-1\right)(F(x, y, z)+(x-1) A(x, y, z)) .
$$

Hence,

$$
A(x, y, z)=\frac{z\left(f_{0}(x, z)-1\right) F(x, y, z)}{1-(x-1) z\left(f_{0}(x, z)-1\right)} .
$$

By substituting the previous expression of $A(x, y, z)$ in relation (3.7), and using relation (3.5), we obtain the required relation (3.6).

From relation (3.6), and using relations (3.2) and (3.5), we have that

$$
\begin{aligned}
F(x, y, z) & =\frac{g_{0}(x, y z)\left(g_{0}(x, z)-y g_{0}(x, y z)\right)}{g_{0}(x, z)-y g_{0}(x, y z)-z(1+(x-1) y z) g_{0}^{2}(x, z) g_{0}(x, y z)+g_{0}(x, z)\left(g_{0}(x, y z)-1\right)} \\
& =\frac{g_{0}(x, z)-y g_{0}(x, y z)}{g_{0}(x, z)-z g_{0}^{2}(x, z)-y\left(1+(x-1) z^{2} g_{0}^{2}(x, z)\right)} \\
& =\left(1-(x-1) z\left(f_{0}(x, z)-1\right)\right) \frac{g_{0}(x, z)-y g_{0}(x, y z)}{1-y} .
\end{aligned}
$$

Since

$$
\left[y^{m}\right]\left(g_{0}(x, z)-y g_{0}(x, y z)\right)= \begin{cases}g_{0}(x, z), & m=0 \\ -\sum_{k \geqslant 0} b_{m-1, k} x^{k} z^{m-1}, & m \geqslant 1\end{cases}
$$

it follows that

$$
\begin{aligned}
f_{m}(x, z) & =\left(1-(x-1) z\left(f_{0}(x, z)-1\right)\right)\left[y^{m}\right]\left(\frac{g_{0}(x, z)-y g_{0}(x, y z)}{1-y}\right) \\
& =\left(1-(x-1) z\left(f_{0}(x, z)-1\right)\right)\left(g_{0}(x, z)-\sum_{n=1}^{m} \sum_{k \geqslant 0} b_{n-1, k} x^{k} z^{n-1}\right) \\
& =\left(1-(x-1) z\left(f_{0}(x, z)-1\right)\right) \sum_{n \geqslant m} \sum_{k \geqslant 0} b_{n, k} x^{k} z^{n} .
\end{aligned}
$$

Corollary 3.2. The number of Grand-Dyck paths with semilength $n$, having $m$ flaws and $k$ occurrences of the string duu is equal to

$$
\left[x^{k} z^{n}\right] f_{m}=b_{n, k}+\sum_{i=1}^{n-m-1} \sum_{j=0}^{k} a_{i, j}\left(b_{n-i-1, k-j}-b_{n-i-1, k-j-1}\right) .
$$

Proof. We first observe that

$$
\left(f_{0}(x, z)-1\right) \sum_{n \geqslant m} \sum_{k \geqslant 0} b_{n, k} x^{k} z^{n}=\sum_{n \geqslant m+1} \sum_{k \geqslant 0} \sum_{j=1}^{n-m} \sum_{j=0}^{k} a_{i, j} b_{n-i, k-j} x^{k} z^{n}
$$


and hence, by Theorem 3.1 it follows that

$$
\left[x^{k} z^{n}\right] f_{m}=b_{n, k}+\sum_{i=1}^{n-m-1} \sum_{j=0}^{k} a_{i, j}\left(b_{n-i-1, k-j}-b_{n-i-1, k-j-1}\right) .
$$

Remark From the previous corollary, it follows that the number of Grand-Dyck paths with semilength $n$ having $m$ flaws and $k$ occurrences of the string $\tau=u d d$ is equal to

$$
b_{n, k}+\sum_{i=1}^{m-1} \sum_{j=0}^{k} a_{i, j}\left(b_{n-i-1, k-j}-b_{n-i-1, k-j-1}\right) .
$$

Corollary 3.3. The number of Grand-Dyck paths with semilength $n$, having $k$ occurrences of the string duu is equal to

$$
(n+1) b_{n, k}+\sum_{i=1}^{n-1}(n-i) \sum_{j=0}^{k} a_{i, j}\left(b_{n-i-1, k-j}-b_{n-i-1, k-j-1}\right) .
$$

For further information concerning the double sequence described in the previous corollary, see A051288 in [22].

\section{References}

[1] Y. Le Borgne, Counting upper interactions in Dyck paths, Sém. Lothar. Combin. 54 (2005/06), Article B54f.

[2] D. Callan, Two bijections for Dyck path parameters, Preprint, (2004), 4pp. http://www.arxiv.org/abs/math.CO/0406381

[3] D. Callan, Some bijections and identities for the Catalan and Fine numbers, Sém. Lothar. Combin. 5 (2006), Article B53e.

[4] D. Callan, A bijection on Dyck paths and its cycle structure, Electron. J. Combin. 14 (2007), \#R28.

[5] K. L. Chung, W. Feller, On fluctuations in coin tossing, Proc. Acad. Sci. USA 35 (1949), 605-608.

[6] A. Denise, R. Simion, Two combinatorial statistics on Dyck paths, Discrete Math. 137 (1995), 155-176.

[7] E. Deutsch, A bijection on Dyck paths and its consequences, Discrete Math. 179 (1998), 253-256.

[8] E. Deutsch, An involution on Dyck paths and its consequences, Discrete Math. 204 (1999), 163-166.

[9] E. Deutsch, Dyck path enumeration, Discrete Math. 204 (1999), 167-202. 
[10] S.-P. Eu, S.-C. Liu and Y.-N. Yeh, Dyck paths with peaks avoiding or restricted to a given set, Stud. Appl. Math. 111 (2003), 453-465.

[11] J. Ma and Y.-N. Yeh, Refinements of $(n, m)$-Dyck paths, European J. Combin., 32 (2011), 92-99.

[12] P. A. MacMahon, Memoir on the Theory of the Partitions of Numbers, Part IV, Philosophical Transactions of the Royal Society of London, Series A 209 (1909), 153-175.

[13] K. Manes, A. Sapounakis, I. Tasoulas and P. Tsikouras, Counting strings at height $j$ in Dyck paths, Journal of Statistical Planning and Inference 141 (2011), 2100-2107.

[14] K. Manes, A. Sapounakis, I. Tasoulas and P. Tsikouras, General results on the enumeration of strings in Dyck paths, The Electronic Journal of Combinatorics 18 (2011), \#P74, 22pp.

[15] T. Mansour, Counting peaks at height $k$ in a Dyck path, J. Integer Seq. 5 (2002), Article 02.1.1.

[16] T. Mansour, Statistics on Dyck paths, J. Integer Seq. 9 (2006), Article 06.1.5.

[17] D. Merlini, R. Sprungoli and M. Verri, Some statistics on Dyck paths, J. Statist. Plann. and Infer. 101 (2002), 211-227.

[18] P. Peart and W.-J. Woan, Dyck paths with no peaks at height $k$, J. Integer Seq. 4 (2001), Article 01.1.3.

[19] A. Sapounakis, I. Tasoulas and P. Tsikouras, Counting strings in Dyck paths, Discrete Math. 307 (2007), 2909-2924.

[20] A. Sapounakis, I. Tasoulas and P. Tsikouras, Some strings in Dyck paths, Australasian J. Combin. 39 (2007), 49-72.

[21] A. Sapounakis, I. Tasoulas and P. Tsikouras, Enumeration of strings in Dyck paths: A bijective approach, Discrete Math. 309 (2009), 3033-3039.

[22] N. J. A. Sloane, The On-Line Encyclopedia of Integer Sequences, (2011), published electronically at http://oeis.org

[23] Y. Sun, The statistic "number of udu's" in Dyck paths, Discrete Math. 287 (2004), 177-186. 